\title{
A safe and simple technique for exchanging central venous catheters
}

\author{
Mitsuo Shimada, Takashi Matsumata, Shigeki Wakiyama, Takashi Maeda, \\ Takashi Kanematsu and Keizo Sugimachi
}

Department of Surgery II, Faculty of Medicine, Kyushu University, 3-1-1 Maidashi, Higashi-ku, Fukuoka 812, Japan

\begin{abstract}
Summary: A safe and simple technique for exchanging central venous catheters is herein described. Our technique is based on a modification of Seldinger's method, in which a new outer sheath is introduced over the previous catheter as a guidewire in order to simplify the exchange of central venous catheters. This technique can be successfully performed by new residents and can be applied to the exchange of clotted catheters.
\end{abstract}

\section{Introduction}

Since the introduction of percutaneous subclavian venepuncture, this venous access has become widely accepted as the route of choice for long-term hyperalimentation and short-term haemodialysis. The subclavian vein catheter can usually be inserted within 20 minutes at the bedside without any difficulty. However, major complications such as pneumothorax, which is related to the insertion technique of the cannula, occurs at a rate of around $4 \%,{ }^{1-3}$ and a misdirection of the catheter tip into the internal jugular vein has been reported to occur in up to $11.5 \%$ of all insertions. ${ }^{4}$ An exchange of catheters is also sometimes necessary due to complications such as either a clotted catheter. Even at a second insertion, the procedure poses the same risks of technical complications. We herein report a safe and simple technique for exchanging the subclavian vein with a modification of Seldinger's technique in order to minimize the abovementioned risks.

\section{Technique}

The Medicut ${ }^{\circledR}$ UK-II catheter kit (Argyl, Tokyo, Japan) has been used for central venous access. The urokinase is immobilized on the catheter to prevent thrombus formation, ${ }^{5-7}$ and the size of sheath, of course, is fit to exactly match the catheter.

The patient is placed in a $20^{\circ}$ Trendelenburg position to reduce the risk of air embolism, and the head is then directed forward or turned slightly to

Correspondence: M. Shimada, M.D.

Accepted: 14 September 1992 the opposite side. The wide area around the insertion site is then strictly prepared with sufficient iodine and draped, while paying special attention to the sterilization of the old catheter itself. A minimal amount of local anesthetic should be used around the insertion site of the catheter. The catheter is clamped above the skin, and about $5 \mathrm{~cm}$ of the clean subcutaneous part of the catheter is withdrawn and then cut at the distal portion of the clamp. Next, a new outer sheath of the puncture needle, which is ideally the same as that used the previous time, is put over the line. At this moment, the catheter itself plays an important role as a guidewire as in Seldinger's technique. While one hand holds onto the external end of the catheter, the other hand grips the outer sheath and advances the outer sheath over the catheter with a rotating movement through the previous subcutaneous tunnel into the intravascular space (Figure 1). After advancing the outer sheath into the vein, the catheter is withdrawn and then a syringe is attached to the sheath. After checking the smooth venous return, a new catheter is inserted into the superior vena cava through the sheath. The entire $15 \mathrm{~cm}$ long catheter should be advanced while turning the patient's head strongly towards the operator in order to prevent any misdirection of the catheter tip into the internal jugular vein. The catheter is then once again anchored at the exit site with a suture of 3-0 silk.

\section{Discussion}

We have performed this type of procedure to replace many catheters, which had generally either 


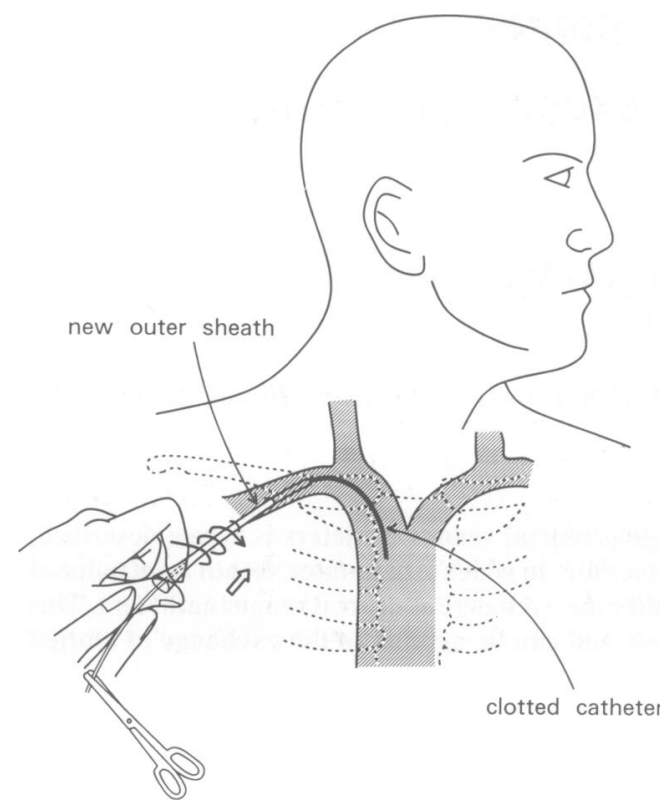

Figure 1 Advancing a new outer sheath over the previous clotted catheter. A new outer sheath is advanced over the previous clotted catheter as a guidewire into the subclavian vein with a rotating movement.

developed clots inside the catheter lumen, or had been misdirected into the internal jugular vein.

The technique for changing catheters using a guidewire has been widely accepted. ${ }^{8-11}$ Pettigrew et al. ${ }^{11}$ advocated that the replacement of catheters over a guidewire is a safe and convenient way of establishing whether sepsis is catheter-related, and recommended that if more than 1,000 colonyforming units of microbial organisms are cultured

\section{References}

1. Bernard, R. \& Starhl, W. Subclavian vein catheterization: a prospective study. I. Non-infectious complications. Ann Surg 1971, 173: 184-190.

2. Ryan, J.A., Abel, R.M., Abbott, W. et al. Catheter complications in total parenteral nutrition: a prospective study of 200 consecutive patients. $N$ Engl J Med 1974, 290: 757-761.

3. Sitzman, J.V., Townsend, T.R., Siler, M.C. \& Bartlett, J.G. Septic and technical complications of central venous catheterization. Ann Surg 1985, 202: 766-770.

4. Padberg, F.T., Jr, Ruggiero, J., Blackburn, G.L. \& Bistrian, B.R. Central venous catheterization of parenteral nutrition. Ann Surg 1981, 193: 264-270.

5. Sugitachi, A. \& Takagi, K. Antithrombogenicity of immobilized urokinase clinical application. Int J Artif Organs 1978, 1: 88-92.

6. Sugitachi, A., Kawahara, T., Kodama, J., Kikkawa, Y. \& Takagi, K. Antithrombogenicity of immobilized urokinase and its clinical significance. Blut 1978, 37: 31-36.

7. Sugitachi, A., Takagi, K., Imaoka, S. \& Kosaki, G. Immobilization of plasminogen activator, urokinase, on Nylon. Thromb Haemost 1978, 39: 426-436. from a catheter tip following a guidewire change, the new catheter should be removed, and if fewer than 1,000 colony-forming units of those are found, the patient is unlikely to have catheterrelated sepsis and another source should be sought. Regarding long-term catheter management, Eyer et al. ${ }^{12}$ stated the general guideline that it is better not to change catheters routinely; to exchange by guidewire, when new catheters are indicated; and, if there is evidence of a skin insertion site infection, to insert a catheter at a new site.

On the other hand, there have been no reports discussing changing catheters using our technique of applying a sheath over the catheter. Our technique can be used even in situations where the catheter is obstructed by a clot inside the catheter lumen. In such situations, the technique of changing catheters using a guidewire loses its efficacy.

Regarding catheter-related sepsis, in principle, we have never applied this technique to exchanging catheters when catheter-related sepsis is suspected. Furthermore, we are used to checking the catheter tip for infection, and the new catheters are removed whenever a culture is positive.

This technique takes only about 5 minutes to carry out, and even new residents can perform the manoeuvre successfully. Moreover, there were no complications related to reinsertion, that is, fon placement outside of the vein or the misdirection ot the catheter.

As our technique uses the previous catheter as a guidewire, it is effective even in situations without fluoroscopy as well as when the previous catheter is determined to be too small for the insertion of a true guidewire. It should not be forgotten, however, that this technique should not be applied in situations where there is the possibility of catheter infection.

8. Padberg, F.T., Ruggiero, J., Blackburn, G.L. \& Bistrian, B.R. Central venous catheterization for parenteral nutrition. Ann Surg 1981, 193: 264-270.

9. Bozzetti, F., Terno, G., Bonfanti, G. et al. Prevention and treatment of central venous catheter sepsis by exchanging via a guidewire. Ann Surg 1983, 198: 48-52.

10. Blewett, J.H., Kyger, E.R. \& Patterson, L.T. Subclavian vein catheter replacement without venipuncture. Arch Surg 1974, 108: 241.

11. Pettigrew, R.A., Lang, S.D.R., Haydock, D.A., Parry, B.R., Bremner, D.A. \& Hill, G.L. Catheter-related sepsis in patients on intravenous nutrition: a prospective study of quantitative catheter cultures and guidewire changes for suspected sepsis. Br J Surg 1985, 72: 52-55.

12. Eyer, S., Brummitt, C., Crossley, K., Siegel, R. \& Cerra, F. Catheter-related sepsis: prospective, randomized study of three methods of long-term catheter maintenance. Crit Care Med 1990, 18: 1073-1079. 